\title{
Construção de um teste objetivo de resistência à frustração
}

\author{
Cristiane Faiad de Moura ${ }^{1}$ \\ Luir Pasquali
}

\begin{abstract}
Resumo
O presente estudo teve como objetivo construir um instrumento de formato objetivo, com base no teste projetivo Picture Frustration Study (PFT) de Rosenzweig, denominado Teste Objetivo de Resistência à Frustração - TORF. Este estudo foi realizado com uma amostra de 555 estudantes universitários de instituições universitárias de Brasília, a partir da aplicação coletiva de um instrumento. A aplicação foi realizada por psicólogos e alunos treinados. As respostas dos sujeitos foram analisadas quanto à ocorrência de cada alternativa. Alguns critérios foram traçados para a escolha do item que compôs a versão final do instrumento. Apesar de não haver indícios na literatura brasileira de uma proposta como esta, este estudo sugere um instrumento promissor a ser trabalhado para uso futuro em seleção de pessoal.
\end{abstract}

Palavras-chave: Frustração; Teste; Forma objetiva.

\section{Construction of objective frustration resistance test}

\begin{abstract}
The aim of this study was to develop an objective instrument, based on the Picture Frustration Study' test (PFT) of Rosenzweig, called Objective Test of Frustration Resistance - TORF. This purpose was carried out with a sample of 555 university students from Brasília Institutions. The students answered an instrument under the supervision of psychologists and students of psychology. The answer was analyse in turns of the alternatives that the instrument allowed. Several criteria were defined in order to choose the alternative for definitive instrument. There is no indication in the brazilian literature of a similar proposal, but the present study suggests such an instrument is very promising for futures personnel selection.
\end{abstract}

Keywords: Frustration; Instrument; Objective form.

\section{O fenômeno da frustração}

Os estudos sobre frustração, até a década de 1960, eram orientados quase que exclusivamente para a verificação da relação entre a frustração e a agressão. Nas últimas décadas, tem-se diminuído a ênfase no estudo da relação entre frustração e agressão mas, mesmo assim, esta relação ainda continua sendo predominante na literatura da área. Pesquisas mais recentes, no entanto, têm ressaltado a frustração como uma característica individual geral que não se relaciona somente com a agressividade, mas também com questões pertinentes à área da saúde e do trabalho (Beghi e colaboradores, 2002; Krejèí, Kvapil \& Semrád, 1996; Shirayama e colaboradores, 2003; Soto, 2005).

Dado que a frustração é vista como fonte de dificuldades e problemas para o indivíduo, espera-se, igualmente, que esta seja a causa do desenvolvimento de mecanismos de defesa ou de superação da própria frustração. Nesta linha de raciocínio, pesquisadores, tais como Rosenzweig (1976), definem tais mecanismos como resistência à frustração.

$\mathrm{Na}$ área do trabalho, assim como na área da saúde, a maneira de lidar ou enfrentar a frustração tem se evidenciado como um dos fatores envolvidos no desempenho e no bem-estar dos trabalhadores (Cebulak, 2001; Fox \& Spector, 1999; Pasquali, Salazar, Freitas, Barbosa \& Roldão, 2002; Swan, 1972; Yates \& Pilai, 1992). O estudo da frustração e de outras características individuais tem sido o tema dominante na investigação do processo de adaptação e desempenho dos profissionais nas organizações (Colquitt, LePine \& Noe, 2000; Costa \& McCrae, 1992; Guion, 1999; Inwald \& Shusman, 1984). Pasquali, Salazar, Freitas, Barbosa e Roldão (2002) e Pasquali, Cabral, Figueira, Rodrigues e Moura (2003), por exemplo, mostram, em suas pesquisas profissiográficas com policiais na área da segurança pública, que, dentre as características individuais necessárias, senão primordiais, para uma adaptação adequada no contexto da estrutura de uma organização e para o bom desempenho de atividades no

Apoio CNPq.

1 Endereço para correspondência:

Universidade de Brasília - ICC Sul, Instituto de Psicologia, Laboratório de Pesquisa em Avaliação e Medida - LabPAM

Sala A1 - 096 - 70910-900 - Brasília-DF

Fone/Fax: (61) 3071114 - Celular: (61) 92138054

E-mail: faiad@unb.br 
trabalho, sobressai-se a resistência à frustração.

Segundo Blume e Naylor (citado por Krejèí e colaboradores, 1996), quando um trabalhador é impedido de realizar uma atividade, sente-se frustrado e pode vir a reagir de uma forma positiva ou não. Krejèí e colaboradores (1996) afirmam que uma das formas negativas da frustração é a agressão. Quando frustradas, as pessoas podem direcionar essa agressão a outras pessoas, ao próprio problema ou a si mesmo.

A importância da agressividade e da resistência à frustração evidencia a necessidade de se avaliarem tais características em todos os indivíduos submetidos a processos seletivos que envolvam profissões públicas e privadas de alto risco ou estresse (p. ex., policiais, professores de escolas públicas, gerentes de empresas). É importante que os psicólogos e outros profissionais, responsáveis por processos de seleção e avaliação, tenham em mãos instrumentos válidos e precisos para mensurar a resistência do indivíduo à frustração. Mesmo assim, e apesar da existência de alguns instrumentos psicológicos disponíveis no mercado, a medida do construto de frustração ainda é deficiente.

Dos testes psicométricos não projetivos utilizados no Brasil para avaliação da personalidade, o único que faz alusão à medida da frustração é o Questionário de 16 Fatores de Personalidade - 16 PF (Catell \& Eber, 1954). Dentre os 16 fatores, o autor propõe um fator de segunda ordem (Q4), que mede a tensão energética do indivíduo e é considerado, também, como uma importante medida da resistência à frustração. Entretanto, além de não ser uma medida direta de frustração, o $16 \mathrm{PF}$ não atende aos requisitos mínimos de padronização, da mesma forma que não possui um manual satisfatório que indique ao psicólogo (resolução 2/2003 do Conselho Federal de Psicologia) a melhor maneira de utilizá-lo como medida do construto frustração. Um outro teste, o Teste de Tolerância à Frustração (TTF), foi validado na Espanha com uma amostra de apenas 32 sujeitos, por Angel, Hernández, Leal e Santacreu (2000), fundamentando-se em uma base comportamental para avaliar a tolerância de sujeitos ao enfrentarem uma tarefa difícil sem recompensas. Além de problemas quanto à sua validade (p. ex., amostra inadequada), o TTF tem outra limitação: a necessidade de se disponibilizar um computador para cada sujeito a fim de que possa ser respondido.

Outros testes que apresentam algum tipo de medida da frustração são os testes projetivos Psicodiagnóstico Miocinético - PMK, Zulliger e Wartegg. O PMK (Mira, 2002) permite uma junção de três fatores (tônus, heteroagressividade e extratensão), denominando-os resistência à frustração. Apesar de apresentar alguma validade de construto, este teste se limita, no entanto, a aplicações individuais, o que o elimina dos grandes processos seletivos coletivos (p. ex., concursos públicos). O teste Zulliger (Vaz, 1998) avalia a resistência à frustração pela análise das respostas categorizadas como "respostas de perspectivas - fator K". Considera-se que indivíduos com alta porcentagem nesse fator não são capazes de tolerar frustração. Esse teste também possui sérios problemas de validade e consistência, além de ter um custo elevado na aplicação.

O teste Wartegg (Kfouri, 1999), que consiste na investigação da personalidade por meio de desenhos feitos com base em estímulos gráficos simples, compreende a análise da resistência à frustração no estudo do campo cinco, campo da energia vital, porém, ainda não é considerado válido como medida da personalidade. Dos testes projetivos, o único que avalia direta e exclusivamente a resistência à frustração é o teste P-F Rosenzweig (PFT), criado na década de 1930 (LaVoie, 1986). Apesar de ter sido considerado inadequado para fins de avaliação psicológica no Brasil (Conselho Federal de Psicologia, 2001; resolução 2/ 2003), pelo Conselho Federal de Psicologia (baseado unicamente na qualidade do manual brasileiro), o teste PFT ainda é o mais utilizado no mundo inteiro para a avaliação da frustração (Angel e colaboradores, 2000). Trata-se, portanto, de um bom ponto de partida para pesquisas que pretendam resultar na construção de novos instrumentos de medida de resistência à frustração.

\section{A medida da frustração segundo Rosenzweig}

Segundo Rosenzweig, o fenômeno da frustração está associado ao da agressão, visto que esta é resultante direta da frustração vivenciada. Para avaliar essa associação, Rosenzweig desenvolveu o Picture Frustration (PF) Study - Teste de Frustração de Rosenzweig - PFT. (LaVoie, 1986; Rosenzweig, 1976, 1978). Esse teste foi criado em 1934 como parte de uma bateria de testes de agressão. A bateria inicial era composta de um teste comportamental, dois testes de reações ante a frustração de múltipla escolha e o teste PFT, tendo sido publicada apenas a forma PFT (Rosenzweig, 1976), dando origem às versões para adultos (acima de 14 anos), para adolescentes (dos 12 aos 18 anos) e para crianças (dos 4 aos 13 anos). A versão adulta foi criada em 1944, revista em 1948, traduzida e adaptada para o português por Eva Nick (Nick, s. d.).

O PFT consiste em 24 desenhos, cada um representando uma situação de frustração que envolve dois personagens. Em cada situação, um personagem 
aparece pronunciando para outro(s) personagem(ns) algumas palavras que descrevem uma situação frustrante. Assim, em cada desenho, um quadradinho vem preenchido com a fala de um dos personagens descrevendo a situação frustrante, enquanto o outro

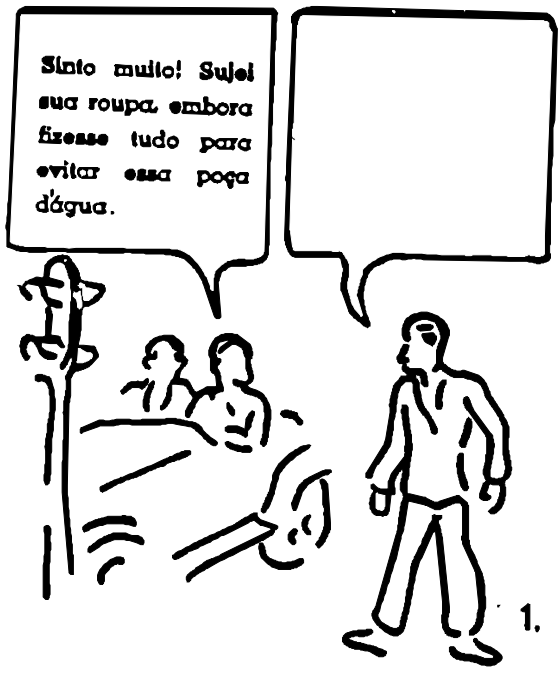

Figura 1 - Reprodução das situações 1 e 2 do teste PFT

O PFT é um teste projetivo, criado com a finalidade de verificar, diante de uma situação frustrante, se o indivíduo reage com uma resposta de tolerância ou intolerância. Entende-se que o indivíduo, consciente ou inconscientemente, identifica-se com o personagem frustrado nas situações apresentadas no teste e se projeta pela resposta que ele elabora (Rosenzweig, Ludwig \& Adelman, 1975). As respostas são medidas da reação dos indivíduos ante uma situação que a maioria das pessoas consideraria como frustrante (como, por exemplo, esbarrar e quebrar o vaso predileto da mãe de uma amiga ou ser totalmente molhado por um carro que passou em uma poça d'água no momento exato em que se anda pela calçada). As situações apresentadas impedem, desapontam, bloqueiam e privam o indivíduo de algo (Rosenzweig, 1976), ou mesmo acusam, insultam ou até incriminam o indivíduo em determinada circunstância.

\section{Categorias de resposta do PFT}

As respostas dadas pelos indivíduos, em cada uma das situações frustrantes, são enquadradas em categorias de respostas definidas no que se refere à direção e ao tipo de agressão. A direção indica para onde a agressão é direcionada pelo indivíduo quando frustrado e se subdivide nas três subcategorias de respostas (cada subcategoria é representada por um símbolo/letra), Psico-USF, v. 11, n. 2, p. 137-146, jul./ dez. 2006 vem em branco e deve ser preenchido pelo outro personagem do desenho, isto é, pela pessoa que está realizando o teste, diante da situação frustrante (ver exemplo das situações 1 e 2 do teste PFT na Figura 1).

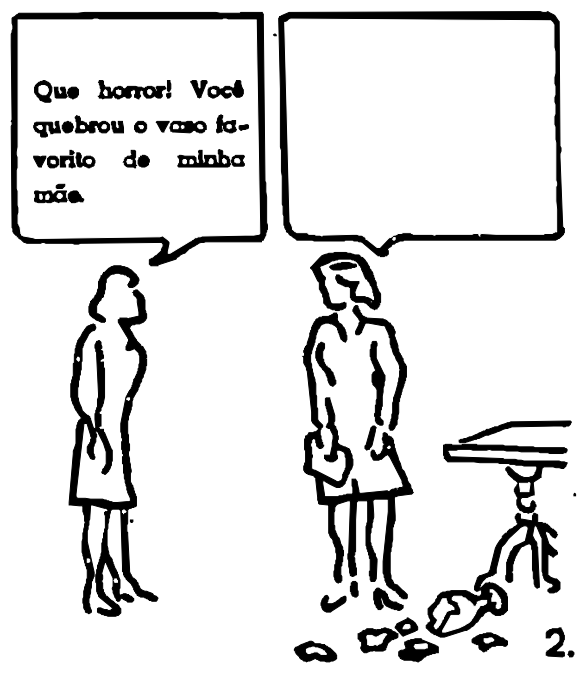

sendo a primeira a resposta extrapunitiva (E), onde a agressão é dirigida para o exterior. Nesse caso, algo ou alguém é culpado pelo indivíduo ter sido frustrado. A segunda é a resposta intrapunitiva (I), na qual a agressão é dirigida para o próprio indivíduo e a terceira é a resposta impunitiva (M), onde a agressão é evitada e a situação frustrante é descrita como sem importância, sem culpa, ou como suscetível de ser melhorada. Aqui, o indivíduo se contenta em esperar que tudo melhore ou, então, conforma-se com o problema.

O tipo de reação manifesta pelo indivíduo indica como o mesmo impulsiona ou mantém a sua agressão, o que, por conseqüência, determina o tipo de sua ação em resposta a estímulos externos. Nota-se, ainda, que o tipo de reação se divide nas categorias de tipo de predominância do obstáculo (OD), no qual o obstáculo que causa a frustração é mencionado e enfatizado pelo sujeito; tipo de defesa do ego (ED), onde o indivíduo lança a culpa sobre outrem ou aceita a responsabilidade ou, ainda, declara que a responsabilidade da situação não cabe a ninguém, e tipo de persistência da necessidade (NP), quando a tendência da resposta é dirigida para a solução do problema inerente à situação frustradora (Dor-Shav \& Mikulincer, 1990; Nick, s. d.; Rosenzweig, 1963, 1976).

A combinação das três direções com os três tipos de agressão produz nove "fatores" que permitem 
a avaliação da resistência à frustração (Rosenzweig e colaboradores, 1975). Além desses fatores, o autor ainda propõe, em algumas situações, a existência de dois fatores extras denominados "Defesa do Ego" (fatores E e I). Um exemplo de resposta de direção extra-agressiva (E) e tipo de dominância do obstáculo (OD), denominado extrapeditivo (E'), seria observado onde o sujeito, diante da situação em que foi quebrado o vaso predileto da mãe de uma amiga, responderia "Puxa! Tinha que ser o vaso que sua mãe mais gostava?" Nesse caso, a situação frustrante é ressaltada na resposta do indivíduo, ou seja, o fato de ter quebrado o vaso predileto da mãe da amiga foi uma situação tão frustrante que foi sublinhada insistentemente pelo indivíduo.

\section{Análise crítica do Teste de Frustração de Rosenzweig}

As críticas ao PFT compreendem dois dos seus aspectos principais, a saber, a sua teoria e a sua forma semiprojetiva.

\section{A teoria}

O teste de frustração de Rosenzweig foi embasado em uma das primeiras teorias fundamentadas para explicar a questão da agressividade, a Teoria da Frustração e Agressão. Essa teoria foi proposta em 1939 por John Dollard, Leonard Doob, Neal Miller, Hobart Mowrer e Robert Sears, ofereceu à comunidade científica uma base teórica para a análise da agressão social, ficando conhecida como a Teoria Clássica da Agressão.

A teoria de Dollard e colaboradores (citado por Berkowitz, 1989) considera que toda agressão seria resultante de uma frustração, e que a frustração aumentaria a instigação para uma agressão. A partir dessa teoria, Rosenzweig (citado por Nick, s. d.) definiu a frustração como um fenômeno que ocorre todas as vezes que o organismo encontra um obstáculo ou impedimento mais ou menos intransponível no caminho, que o conduz à satisfação de qualquer necessidade vital. Ela ocorre, ainda, quando o organismo é submetido à ausência ou presença de um estímulo que seja agradável, ou à ausência de um objeto externo suscetível de satisfazer a pulsão. Nesta última concepção, inclusive, as propostas de Rosenzweig se aproximam bastante das idéias de Freud (Laplanche \& Pontalis, 1998).

Rosenzweig (1945) propôs um esquema de análise, diferenciando a frustração em dois tipos básicos, primária e secundária, sendo esta última a de interesse deste trabalho. A frustração secundária estaria relacionada à natureza do obstáculo ou fonte da frustração e nesta visão, a frustração estaria ligada a um tipo de defesa do ego, o que, segundo a psicanálise, seriam respostas em defesa da ameaça a tal ego. Ainda de acordo com Rosenzweig (1945), o indivíduo, quando frustrado, reagiria de diferentes maneiras como forma de defesa, agredindo ou não agredindo, direcionando essa agressão a ele mesmo, a outrem, ou simplesmente evitando alguma agressão.

As teorias mais atuais afirmam que a relação entre agressão e frustração existe, mas que a agressão é, na verdade, uma das possíveis reações que o indivíduo pode apresentar quando frustrado. A teoria atual que melhor esclarece essa relação é a Teoria Neoassociacionista de Berkowitz (1962, 1989, 1990, 1993, 1998; Berkowitz, Jaffee, Jo \& Tróccoli, 2000), que objetivou não substituir, mas complementar a Teoria Clássica, utilizando-se de uma perspectiva cognitivista. Segundo Berkowitz (1990), a frustração leva à reação agressiva, dependendo da interpretação que o indivíduo faz da situação (Hoffmman \& Legal, 2003).

\section{A forma}

Apesar de ser um dos poucos testes existentes para avaliar a resistência à frustração, o PFT não se encontra na lista dos mais comumente utilizados nos diversos países. LaVoie (1986) considera que, apesar dos mais de 500 estudos publicados com o uso do teste PFT, a maior parte dos seus problemas decorre do seu caráter projetivo. Dentre as críticas ao teste PFT ressalta-se o problema da norma utilizada nos estudos de padronização para diversos países, bem como a característica ultrapassada dos desenhos, construídos de acordo com a realidade da década de 30, e o procedimento de inquérito inviável para aplicações coletivas. Nota-se, ainda, como críticas, a falta de concordância entre os avaliadores no procedimento de correção, o problema na avaliação do nível de consciência dos indivíduos ao responderem o teste e, ainda mais evidente, a necessidade de se atualizar a teoria que embasa o instrumento. Bjerstedt (1965) propõe, como solução para tais críticas, a realização de mais pesquisas considerando-se aspectos tais como tamanho da amostra e inconsistência dos itens. Todas essas críticas atribuídas ao PFT, e aos instrumentos projetivos em geral, sinalizam certas incompatibilidades entre o uso, a prática e a medida.

\section{Proposta do presente estudo}

As críticas levantadas à teoria e à forma do PFT são os pontos primordiais que fundamentam a proposta apresentada no presente estudo, na construção de um novo teste de resistência à frustração. Do ponto de vista 
teórico, a criação de um novo teste trouxe como proposta uma adequação teórica mais atual e compreensível sobre a frustração. Optou-se por utilizar as mesmas categorias de reação à frustração criadas por Rosenzweig, embora a grande diferença esteja na compreensão entre os fenômenos de frustração e agressão de acordo com a teoria proposta por Berkowitz (1962). $\mathrm{Na}$ análise da forma do teste, algumas considerações merecem ser destacadas quanto a suas limitações e adequações de uso.

Em face das possibilidades do uso do teste, coube questionar não a sua capacidade de produzir riqueza de dados, mas as suas possibilidades de aplicabilidade e codificação para uma amostra coletiva, bem como uma necessária análise de sua fundamentação teórica dada à importância deste construto na avaliação, por exemplo, de policiais. LaVoie (1986) considera que os dados do PFT são ricos quando aliados a outras técnicas de avaliação, mas não se encontra na literatura o uso deste instrumento na área de seleção de pessoal, talvez em razão do seu caráter projetivo, porém os estudos mostram apenas seu uso nas temáticas da área da psicologia social.

Testes como o PFT, de origem projetiva, caracterizam-se por apresentar tarefas relativamente "não estruturadas", que permitem aos indivíduos uma variedade quase que ilimitada de possíveis respostas. Presume-se que a maneira como o indivíduo interpreta o teste, ou a forma como ele estrutura a situação, reflita aspectos fundamentais de seu funcionamento psicológico, o que corrobora sua utilização pela riqueza dos dados (Anastasi \& Urbina, 2000).

Os testes projetivos, apesar das aparentes análises complexas dos indivíduos, trazem certa limitação em seus procedimentos de codificações das respostas dadas. Estas, normalmente, são analisadas com base no quadro de referência do psicólogo avaliador, o que implica o envolvimento da sua subjetividade, ao mesmo tempo em que se busca um procedimento padronizado de aplicação, pontua-ção e interpretação. Esses dois últimos aspectos são os responsáveis pelas grandes dificuldades da utilização dos testes projetivos em seleção de pessoal, por exemplo.

Existem muitos questionamentos entre o uso das técnicas psicometristas versus impressionistas (Cronbach, 1996; Pasquali, 2001), principalmente no que tange aos processos de avaliação. A objetividade das técnicas psicométricas, caracterizada por tarefas padronizadas, correção, apuração mecânica e interpretação realizada segundo normas, traz a segurança de um trabalho isonômico, mas fortemente criticado pelo mapeamento simplificado dos indivíduos. Já as técnicas impressionistas, caracteriza-das por trabalharem amplamente com a subjetividade, tarefas pouco ou nada estruturadas, respostas que deixam margem para interpretações subjetivas do próprio avaliador, trazem a riqueza dos dados e, também, a inexatidão da avaliação como um todo.

No que se refere à avaliação psicológica na seleção de pessoal, por exemplo, cabe avaliar não a melhor proposta, pois cada uma possui sua característica intrínseca, mas sim o melhor método para um processo de seleção de pessoal. Ou seja, em outras palavras, um método que possa avaliar, de forma isonômica, cada um dos candidatos, e que traga o rigor dos padrões de validade e qualidade.

Segundo Noronha e Vendramini (2003), encontrar técnicas projetivas e testes de personalidade que não apresentem estudos de padronização, validade e precisão não é uma surpresa na literatura. As autoras afirmam que, apesar dessa temática ser muito discutida na comunidade científica, o maior problema se encontra nas características específicas que diferenciam as técnicas projetivas das não-projetivas. Anzieu (1979) defende que essa diferença exige um remanejamento dos padrões de análises, propondo que essas técnicas devam ser tratadas diferentemente em seus conceitos e medidas. Esta mesma idéia é corroborada por MacFarlane e Tuddenham (1978), que compreendem que os testes projetivos dificultam a integração dos métodos e critérios psicométricos. Nesse sentido, Anastasi e Urbina (2000) propõem, como solução para tais contradições, a construção de formas paralelas desses testes, mais criteriosa e válida.

Dado o grau de seriedade do trabalho realizado em avaliação psicológica, o ideal, portanto, é que seja criado um teste que una a riqueza de manifestações espontâneas, típicas dos testes projetivos, que apresente um bom fundamento teórico para sua aplicação coletiva e, igualmente, a objetividade e padronização de aplicação e correção necessárias a procedimentos como a avaliação em seleção de pessoal. O teste projetivo PFT parece ser a escolha mais apropriada para ponto de partida de tal teste, pois pode ser transformado e atualizado naquilo em que apresenta problemas ou defasagem por conta de uma concepção original parcialmente ultrapassada.

O PFT tem sido um bom instrumento para a coleta, sistematização e categorização de boas amostras de respostas verbais possíveis - não necessariamente universais ou permanentes -, apresentadas pelos indivíduos quando confrontados com situações que provocam frustrações; conforme discutido anteriormente, seus principais problemas se situam, além da teoria que o fundamenta, na captura e análise das reações dos respondentes. Propôs-se, então, a construção de um teste objetivo que tome por base a 
versão original do teste projetivo de Resistência à Frustração P-F Rosenzweig. Este é o objetivo geral da presente pesquisa: a construção e validação de um teste objetivo de resistência à frustração com base no P-F Rosenzweig.

\section{Método}

Para a elaboração do Teste Objetivo de Resistência à Frustração (TORF), foi criada uma primeira versão baseada no teste projetivo PFT. Essa versão continha as 24 situações do PFT acompanhadas por sentenças representando todas as respostas ligadas às possíveis reações à frustração. As sentenças foram retiradas das respostas dadas ao teste PFT em seleções anteriores, foram elaboradas por 6 juízes (especialistas no teste) e retiradas do manual do teste. Essas sentenças foram agrupadas em oito conjuntos (impressos em cadernos separados), correspondendo cada um às seguintes situações do teste: grupo 1 (situações 1, 2 e 3); grupo 2 (situações 4, 5 e 6); grupo 3 (situações 7, 8 e 9); grupo 4 (situações 10, 11 e 12); grupo 5 (situações 13, 14 e 15); grupo 6 (situações 16, 17 e 18); grupo 7 (situações 19, 20 e 21) e grupo 8 (situações 22, 23 e 24). Todas as sentenças foram codificadas e agrupadas de forma a identificar, para cada uma das 24 situações do teste, uma variedade de respostas que abarcasse a codificação proposta pelo teste original, isto é, as categorias: E, E', e, I, I', i, M, M', m, I, E.

\section{Participantes}

Participaram deste estudo 555 estudantes voluntários, sendo 362 mulheres $(65,2 \%)$ e 165 homens (29,7\%), com idade média de 25,08 anos (DP $=7,34$ ). Quanto ao nível de escolaridade, a maioria $(\mathrm{N}=368$; $66,3 \%$ ) tinha nível superior incompleto. A amostra foi composta de estudantes de ensino médio da rede pública do Distrito Federal e estudantes universitários da rede particular e pública. Os participantes foram subdivididos em 8 grupos escolhidos aleatoriamente. Cada grupo respondeu a 1 caderno, cada sujeito respondendo a apenas 1 caderno.

\section{Instrumento}

Os oito cadernos continham as 24 situações do teste original PFT, acompanhadas de frases representativas de cada categoria do teste. Em cada categoria foi apresentado um enunciado solicitando ao participante que fizesse uma escolha, dentre 5 alternativas, daquela que representasse melhor seu modo de falar (agressivo, impunitivo, intrapunitivo). Assim, por exemplo, para a situação 1 do instrumento havia até 11 possibilidades de respostas representando cada uma das categorias: E, E', e, I, I', i, M, M',m, I, E, tendo cada uma delas 5 alternativas de escolha (modos de falar). O participante deveria escolher uma dentre as cinco alternativas para cada uma das situações. Dessa forma, foram definidas, para cada uma das 24 situações, conjuntos de respostas objetivas. Cada situação continha entre 7 e 11 alternativas de respostas, totalizando-se 209 itens ou variáveis.

É preciso notar que algumas situações foram acompanhadas de menos que 11 categorias ou dimensões, porque, segundo as definições de Rosenzweig e colaboradores (1975), logicamente elas não fazem sentido, tais como, a resposta ' $m$ ' na situação 2 do teste, o que implicaria dizer que, com o tempo, o vaso predileto da mãe da amiga sairia consertado.

\section{Procedimento}

Os respondentes foram abordados em sala de aula, com a permissão antecipada de cada professor. A aplicação foi coletiva e realizada por psicólogos, alunos de psicologia e pelos próprios professores, seguindo uma folha explicativa com todas as instruções que deveriam ser dadas antes da aplicação. Cada aplicador apresentou a atividade como uma pesquisa realizada pelo Laboratório de Pesquisa em Avaliação e Medida (LabPAM) do Instituto de Psicologia da UnB sem identificar a temática da pesquisa. Os cadernos foram distribuídos de forma aleatória. Os participantes levaram de 10 a 30 minutos para responder o instrumento.

\section{Análise de dados}

As respostas dos sujeitos foram analisadas de acordo com a ocorrência de cada alternativa escolhida. Os critérios de seleção das alternativas que compuseram o instrumento final foram: (1) a resposta que obteve maior freqüência na escolha dos respondentes; (2) em caso de empate nas freqüências de respostas, a alternativa foi definida por um juiz. Dessa forma, das cinco alternativas de respostas para cada categoria, apenas uma foi escolhida para melhor definir cada situação.

\section{Resultados}

Sobre as escolhas dos participantes em cada uma das situações, observou-se uma grande variabilidade nas respostas. A Tabela 1 apresenta a alternativa da categoria que obteve maior percentual de resposta pelos participantes, ou seja, a alternativa que melhor representou seu modo de falar nas situações de 21 a 24 . A primeira coluna apresenta as categorias de cotação da resposta do teste original; a segunda subdivide-se no percentual de cada uma das respostas. A alternativa de maior percentual, escolhida pelos participantes, foi identificada em itálico.

Psico-USF, v. 11, n. 2, p. 137-146, jul./ dez. 2006 
Tabela 1 - Percentual de escolha de alternativas nas situações 21 a 24 do teste

\begin{tabular}{|c|c|c|c|c|c|c|c|c|c|c|c|c|c|}
\hline \multirow{2}{*}{$\begin{array}{l}\text { Categorias/ } \\
\text { Dimensões }\end{array}$} & \multicolumn{6}{|c|}{ Frase da Situação $21(\mathrm{~N}=70)$} & \multirow{2}{*}{$\begin{array}{l}\text { Categorias/ } \\
\text { Dimensões }\end{array}$} & \multicolumn{6}{|c|}{ Frase da Situação $22(\mathrm{~N}=72)$} \\
\hline & $\mathrm{a}$ & $\mathrm{b}$ & $\mathrm{c}$ & $\mathrm{d}$ & $\mathrm{e}$ & $\mathrm{f}$ & & A & $\mathrm{b}$ & $\mathrm{c}$ & $\mathrm{d}$ & $\mathrm{e}$ & $\mathrm{f}$ \\
\hline $\mathrm{E}$ & 14,3 & 27,1 & 25,7 & 4,3 & 20,0 & 7,1 & $\mathrm{E}^{\prime}$ & 23,6 & 13,9 & 8,3 & 27,8 & 19,4 & 6,9 \\
\hline$\underline{E}$ & 20,0 & 24,3 & 5,7 & 12,9 & 34,3 & 1,4 & $\mathrm{E}$ & 13,9 & 38,9 & 8,3 & 25,0 & 6,9 & 4,2 \\
\hline I' & 21,4 & 10,0 & 25,7 & 20,0 & 18,6 & 1,4 & e & 52,8 & 5,6 & - & 13,9 & 27,8 & - \\
\hline$\underline{I}$ & 20,0 & 25,7 & 32,9 & 7,1 & 7,1 & 4,3 & I' & 18,1 & 25,0 & 8,3 & 18,1 & 23,6 & 6,9 \\
\hline I & 25,7 & 22,9 & 10,0 & 28,6 & 8,6 & 1,4 & I & 18,1 & 18,1 & 15,3 & 27,8 & 19,4 & 1,4 \\
\hline $\mathrm{i}$ & 10,0 & 15,7 & 22,9 & 35,7 & 10,0 & 2,9 & $M^{\prime}$ & 40,3 & 13,9 & 19,4 & 13,9 & 12,5 & - \\
\hline$M^{\prime}$ & 14,3 & 25,7 & 35,7 & 5,7 & 4,3 & 1,4 & M & 27,8 & 12,5 & 27,8 & 22,2 & 9,7 & - \\
\hline M & 4,3 & 40,0 & 8,6 & 2,9 & 1,4 & 1,4 & $\mathrm{~m}$ & 22,2 & 12,5 & 47,2 & 4,2 & 12,5 & 1,4 \\
\hline Categorias/ & \multicolumn{6}{|c|}{ Frase da Situação 23 (N =72) } & Categorias/ & \multicolumn{6}{|c|}{ Frase da Situação $24(\mathrm{~N}=72)$} \\
\hline Dimensões & $\mathrm{a}$ & $\mathrm{b}$ & $\mathrm{c}$ & $\mathrm{d}$ & e & $\mathrm{f}$ & Dimensões & $\mathrm{a}$ & $\mathrm{b}$ & $\mathrm{c}$ & $\mathrm{d}$ & $\mathrm{e}$ & $\mathrm{f}$ \\
\hline $\mathrm{E}^{\prime}$ & 33,3 & 12,5 & 6,9 & 26,4 & 13,9 & 6,9 & $\mathrm{E}^{\prime}$ & 38,9 & 19,4 & 18,1 & 5,6 & 11,1 & 6,9 \\
\hline $\mathrm{E}$ & 6,9 & 34,7 & 13,9 & 8,3 & 31,9 & 2,8 & $\mathrm{E}$ & 34,7 & 30,6 & 13,9 & 5,6 & 8,3 & 5,6 \\
\hline e & 34,7 & 9,7 & 29,2 & 23,6 & 2,8 & - & e & 31,9 & 8,3 & 2,8 & 23,6 & 31,9 & - \\
\hline I' & 25,0 & 15,3 & 9,7 & 15,3 & 33,3 & 1,4 & I & 15,3 & 11,1 & 15,3 & 47,2 & 11,1 & - \\
\hline I & 5,6 & 18,1 & 8,3 & 41,7 & 23,6 & 2,8 & $\mathrm{i}$ & 27,8 & 9,7 & 12,5 & 31,9 & 18,1 & - \\
\hline $\mathrm{i}$ & 30,6 & 26,4 & 13,9 & 15,3 & 13,9 & - & $M^{\prime}$ & 25,0 & 29,2 & 8,3 & 8,3 & 29,2 & - \\
\hline M' & 29,2 & 15,3 & 5,6 & 25,0 & 3,6 & 0,4 & M & 11,1 & 40,3 & 12,5 & 11,1 & 25,0 & - \\
\hline M & 7,2 & 9,7 & 0,8 & 6,7 & 2,8 & 2,8 & & & & & & & \\
\hline $\mathrm{m}$. & 0,8 & 2,5 & 3,6 & 3,9 & 7,8 & 1,4 & & & & & & & \\
\hline
\end{tabular}

Os números em itálico identificam as frases de maior freqüência de escolha.

Os dados mostrados representam apenas as situações 21 a 24, a título de exemplo da análise.

Os dados da tabela acima demonstram que, para cada situação do teste, uma das alternativas obteve maior freqüência, sendo esta escolhida como a resposta que melhor representa a categoria. Essas respostas deram origem ao Teste Objetivo de Resistência à Frustração - TORF.

A estrutura de análise do TORF segue o modelo proposto por Rosenzweig em 1930, de distinguir direção e tipos de agressão, diferenciando-se desse autor pelo fato do novo instrumento ter forma objetivada das respostas dos sujeitos. As direções de agressão e as dimensões avaliadas, com seus respectivos símbolos, são as seguintes:

A - Direção da agressão:

(1) Extrapunitivo - E, o indivíduo, quando frustrado, direciona sua agressão para o exterior, agredindo alguém ou algo; (2) Intrapunitivo, o indivíduo tende a culpar a si mesmo e assumir a culpa quando frustrado; (3) Impunitivo, o indivíduo, quando frustrado, evita qualquer tipo de comportamento agressivo e tende a afastar ou diminuir o efeito da frustração.

\section{B - Dimensões:}

(1) Agressividade extrapunitiva com dominância do obstáculo - E' (AGREOD), o indivíduo age de forma agressiva e enfatiza, em sua resposta, que a situação é de fato frustrante;
(2) Agressividade extrapunitiva com dominância na defesa do ego - E (AGREED), o indivíduo se defende, agredindo outrem quando frustrado;

(3) Agressividade extrapunitiva com dominância na defesa do ego com desculpa - E (AGREEDT), o indivíduo agride o outro quando frustrado, mas tenta se desculpar por estar sendo agressivo, como forma de defender-se;

(4) Agressividade extrapunitiva com persistência da necessidade - e (AGRENP), o indivíduo age de forma a exigir que alguém tome alguma providência sobre a situação frustrante ou que dê alguma solução à mesma; (5) Agressividade intrapunitiva com dominância no obstáculo - I' (AGRIOD), o indivíduo se sente responsável pelo ocorrido, enfatizando em sua resposta que ele provocou uma situação de fato frustrante;

(6) Agressividade intrapunitiva com dominância do ego I (AGRIED), o indivíduo assume a responsabilidade pela situação frustrante e lamenta o ocorrido. A agressão é voltada para ele mesmo, podendo ser entendida como um tipo de auto-agressão ou autocrítica;

(7) Agressividade intrapunitiva com dominância na defesa do ego - I (AGRIEDT), o indivíduo se culpa pelo ocorrido, mas tenta desculpar-se ou explicar-se pelo fato, como forma de se defender;

(8) Agressividade intrapunitiva com persistência da necessidade - i (AGRINP), o indivíduo assume a culpa e se propõe a corrigir a situação; 
(9) Agressividade impunitiva com dominância no obstáculo - M' (AGRMOD), o indivíduo tenta minimizar a situação frustrante, evitando a agressão, chegando a negar que a situação seja de fato frustrante;

(10) Agressividade impunitiva com dominância do ego M (AGRMED), o indivíduo exime o outro, o ambiente ou a ele mesmo de qualquer culpa sobre a situação frustrante, evitando a ocorrência de agressão;

(11) Agressividade impunitiva com persistência da necessidade - m (AGRMNP), o indivíduo afirma não haver frustração e que, de qualquer modo, a situação se resolverá por si mesma.

O TORF é composto de 24 situações representadas por desenhos, e cada uma contém de 7 a 11 possibilidades de resposta, caracterizando, cada uma delas, as dimensões do teste. Neste sentido, a estrutura do TORF diferenciou-se do teste de Rosenzweig, por seu caráter objetivo nas respostas.

\section{Discussão}

O objetivo deste estudo foi construir um teste de formato objetivo, com base no teste PFT. A seguir serão discutidos os dados obtidos neste estudo.

O TORF é baseado na estrutura proposta por Rosenzweig e cols. (1975), embora modificada pela visão teórica do neoassociassionismo (Berkowitz, 1962; 1989) no entendimento do fenômeno da frustração.

Os dados mostram que o teste construído possibilita a aplicação coletiva e codificação dos dados por um crivo, dado o caráter objetivo do mesmo. A composição final deste instrumento teve as alternativas de cada situação (representando cada uma 1 categoria ou dimensão diferente) distribuídas de forma aleatória, para que não mantivessem um padrão seqüencial sistemático de respostas.

Este estudo apresenta uma validade de conteúdo do teste, já que no levantamento de todas as possibilidades de resposta do teste (Pasquali, 2003) constitui-se uma amostra representativa do construto de acordo com o universo de comportamentos delimitados pela teoria.

Observa-se, que em alguns casos, a diferença entre as porcentagens foi mínima. Esse dado sugere uma possível criação de versões alternativas do instrumento, em pesquisas futuras. Pode-se concluir que, apesar do objetivo ter sido alcançado, alguns problemas dos parâmetros psicométricos do teste deverão ser analisados, com base na análise de validade e precisão do instrumento.

Não há indícios, na literatura brasileira, de uma proposta sobre a construção de um teste de frustração objetivado. Este estudo, além de suprir a falta de um teste de frustração objetivado válido para o mercado, possibilita, também, contribuições aos estudos teóricos do teste PFT. Afinal, o presente estudo responde a alguns dos questionamentos levantados sobre a validade da proposta de Rosenzweig, que embasa a estrutura do TORF e atende à necessidade, segundo Bjerstedt (1965), de se trabalharem diferentes formas de administração e correção do PFT. Além disso, compreende um estudo que enfrenta as críticas dos critérios psicométricos atribuídos a testes projetivos, como o PFT que, segundo Anastasi e Urbina (2000), podem ser solucionados com base na construção de formas paralelas do teste.

Nesse sentido, a escolha da forma do TORF foi determinada a partir da análise dos estudos realizados com o teste PFT, além das críticas atribuídas à forma e à teoria do teste, resultando na criação de um formato diferenciado, embora com a mesma estrutura das situações ou desenhos. Assim, apesar de ser um estudo inovador na literatura, ele constitui uma tentativa de se validar um novo instrumento, inclusive por permitir a comparação dos resultados obtidos com a proposta de Rosenzweig, bem como com os estudos realizados com o PFT.

\section{Limitações do estudo}

Quanto à estrutura do teste, o uso das mesmas situações contidas no teste PFT para configuração do teste TORF se justificaram ao longo deste trabalho, dado o objetivo de se avaliar e testar a proposta feita por Rosenzweig em 1930. Contudo, sugere-se que futuras pesquisas sejam realizadas com situações que retratem a atualidade ou, até mesmo, retratem situações mais específicas do trabalho de policiais, por exemplo.

\section{Agradecimentos}

Agradecemos à valiosa colaboração do prof. dr. Bartholomeu T. Tróccoli para o andamento do projeto.

\section{Referências}

Anastasi, A. \& Urbina, S. (2000). Testagem psicológica. Porto Alegra: ArtMed.

Angel, L. M., Hernández, J. M., Leal, O. G. \& Santacreu, J. (2000). Un test informatizado para la evaluación de la tolerancia a la frustración. Anales de Psicología, 16(2), 143-155.

Anzieu, D. (1979). Os métodos projetivos. Rio de Janeiro: Campus.

Beghi, E., Spagnoli, P., Airoldi, L., Fiordelli, E., Appollonio, I., Bogliun, G., Zardi, A., Paleari, F., Psico-USF, v. 11, n. 2, p. 137-146, jul./ dez. 2006 
Gamba, P., Frattola, L. \& Da Prada, L. (2002). Emotional and affective disturbances in patients with epilepsy. Epilepsy \& Behavior, 3(3), 255-261.

Berkowitz, L. (1962). The frustration-agression bypothesis. Aggression: A social psychological analysis. New York: MacGraw-Hill.

Berkowitz, L. (1989). The frustration-aggression hypothesis: Examination and reformulation. Psychological Bulletin, 106, 59-73.

Berkowitz, L. (1990). On the formation and regulation of anger and aggression: A cognitiveneoassociationistic analysis. American Psychologist, 45, 494-503.

Berkowitz, L. (1993). Pain and aggression: Some findings and implications. Motivation and Emotion, 17, 277-293.

Berkowitz, L. (1998). Affective aggression: The role of stress, pain and negative affect. Em R. G. Geen \& E. Donnerstein (Orgs.). Human aggression: Theories, research, and implications for social policy (pp. 49-72). San Diego, CA: Academic Press.

Berkowitz, L., Jaffee, S., Jo, E. \& Tróccoli, B. T. (2000). On the correction of feeling-induced judgmental biases in feeling and thinking. Em J. Forgas (Org.). Feeling and thinking: The role affect in social cognition (pp. 131-152). Cambridge University: New York.

Bjerstedt, A. (1965). Rosenzweig picture-frustration study. Em O. K. Buros (Org.). The sixth mental measurements yearbook (pp. 511-515). Highland Park, NJ: The Gryphn Press.

Catell, R. B. \& Eber, H. W. (1954). Manual de psicologia aplicada $16 \mathrm{PF}$ : formas $A$ e B (adultos) (E. M. Andrade \& D. G. Alves, tradução e adaptação brasileira). Rio de Janeiro: Cepa. (Original publicado em 1955).

Cebulak, W. (2001). Fairness, job frustration, and moral dilemmas in policing that impact police effectiveness. Journal of Police and Criminal Psychology, 16, 48.

Colquitt, J. A., LePine, J. A. \& Noe, R. A. (2000). Toward and integrative theory of training motivation: A meta-analytic path analysis of 20 years of research. Journal of Applied Psychology, 85(5), 678-707.

Conselho Federal de Psicologia (2001, dezembro). CFP aprova duas novas resoluções. Jornal do Federal, 5.

Conselho Federal de Psicologia (2003). Resolução $n^{\circ} 2$. Define e regulamenta o uso, a elaboração e a comercialização de testes psicológicos e revoga a Resolução CFP no 25/2001.
Costa, P. T., Jr. \& McCrae, R. R. (1992). Four ways five factors are basic. Personality and Individual Differences, 12, 653-665.

Cronbach, L. J. (1996). Fundamentos da testagem psicológica. (C. A. S. Neto \& M. A. V. Veronese, tradução). Porto Alegre: Artes Médicas. (Original publicado em 1990).

Dollard, J., Doob, L., Miller, N., Mowrer, O. \& Sears, R. (1939). Frustration and aggression. New Haven, CT: Yale University Press.

Dor-Shav, N. K. \& Mikulincer, M. (1990). Learned helplessness, causal attribution, and response to frustration. The Journal of General Psychology, 11(1), 4758.

Fox, S. \& Spector, P. E. (1999). A model of work frustration-aggression. Journal of Organization Behavior, 20(6), 915.

Guion, R. M. (1999). Some virtues of dissatisfaction in the science and practice of personnel selection. Human Resource Management Review, 8, 351-365.

Hoffmman, M. H. \& Legal, E, J. (2003). Comportamento agressivo e acidentes no trânsito em comportamento bumano no trânsito. São Paulo: Casa do Psicólogo.

Inwald, R. \& Shusman, E. (1984). The IPI and MMPI as predictors of academy performance for police recruits. Journal of Police Science and Administration, 12, $1-11$.

Kfouri, N. J. (1999). Wartegg: da teoria à prática. São Paulo: Vetor.

Krejèí, P., Kvapil, J. \& Semrád, J. (1996). The relation between job satisfaction, job frustration and narcissism and attitudes towards professional ethical behavior among police officers. Em College of Police and Security Studies - Slovenia. Policing in Central and Eastern Europe: comparing firsthand knowledge with experience from the West [On-line]. Disponível em: <http://www.unojust.org>.

Laplanche, J. \& Pontalis, J. B. (1998). Vocabulário da psicanálise. São Paulo: Martins Fontes.

LaVoie, A. L. (1986). Rosenzweig picture-frustration study. Em D. J. Keyser \& R. C. Sweetland (Orgs.). Test critiques (v. 14, pp. 388-395). Kansas: Test Corporation of America.

MacFarlane, J. W. \& Tuddenham, R. D. (1978). Dificuldades para a validação das técnicas projetivas. Em H. H. Anderson \& G. L. Anderson (Orgs.). Técnicas projetivas do diagnóstico psicológico (pp. 41-68). São-Paulo: Mestre. 
Mira, A. M. G. (2002). PMK: psicodiagnóstico miocinético. São Paulo: Vetor.

Nick, E. (s. d.). Manual do teste de frustração: forma para adulto. (S. Rosenzweig, Trad.) Rio de Janeiro: CEPA.

Noronha, A. P. P. \& Vendramini, C. M. M. (2003). Parâmetros psicométricos: estudo comparativo entre testes de inteligência e de personalidade. Psicologia: Reflexão e Crítica, 16(1), 177-182.

Pasquali, L. (2001). Técnicas de Exame Psicológico - TEP. Manual. Brasília: Casa do Psicólogo.

Pasquali, L. (2003). Psicometria: Teoria dos testes na psicologia e na educação. Rio de Janeiro: Vozes.

Pasquali, L., Salazar, A. A., Freitas, L. C. O., Barbosa, B. \& Roldão, A. C. (2002). Profissiografia do cargo de policial rodoviário da Polícia Rodoviária Federal. Manuscrito não publicado, LabPAM, Universidade de Brasília.

Pasquali, L., Cabral, A. R., Figueira, K. S., Rodrigues, M. M. M. \& Moura, C. F. (2003). Profissiografia do cargo de agente da polícia civil do Distrito Federal. [On-line]. Disponível em: www.mj.gov.br/senasp/monografias.

Rosenzweig, S. (1945). The picture association method and its application in a study of reactions to frustration. Journal of Personality, 14, 3-23.

Rosenzweig, S. (1963). Validity of the Rosenzweig Picture-Frustration Study with felons and deliquents. Journal of Consulting Psychology, 27(6), 535-536.

Rosenzweig, S. (1976). Aggressive behavior and the Rosenzweig Picture-Frustration (P-F) Study. Journal of Clinical Psychology, 32(4), 885-891.
Rosenzweig, S. (1978). An investigation of the realiability of the Rosenzweig Picture-Frustration (PF) Study children's form. Journal of Personality Assessment, 42, 483-488.

Rosenzweig, S., Ludwig, D. J. \& Adelman, S. (1975). Retest reliability of the Rosenzweig PictureFrustration Study and similar semiprojective techniques. Journal of Personality Assessment, 39, 3-12.

Shirayama, M., Shirayama, Y., Iida, H., Kato, M., Kajimura, N., Watanabe, T., Sekimoto, M., Shirakawa, S., Okawa, M. \& Takahashi, K. (2003). The psychological aspects of patients with delayed sleep phase syndrome (DSPS). Sleep Medicine, 4(5), 427-433.

Soto, E. (2005). Comportamento organizacional: o impacto das emocões. São Paulo: Pioneira Thompson Learning.

Swan, H. F. (1972). Personnel induced frustration. The Canadian Chartered Accountant, 100(4), 48.

Vaz, C. E. (1998). Z-teste: forma coletiva. São Paulo: Casa do Psicólogo.

Yates, D. L. \& Pilai, V. K. (1992). Frustration and strain smong fort worth police officers. Sociology and Social Research, 76(3), 145.

Sobre os autores:

Cristiane Faiad de Moura é psicóloga, mestre e doutoranda em Psicologia Social e do Trabalho. Atua na área de construção de instrumentos pelo Laboratório de Pesquisa em Avaliação e Medida, como consultora em avaliação psicológica e como professora de pós-graduação em Gestão de Pessoas.

Luiz Pasquali é doutor, professor emérito pela Universidade de Brasilia, coordenador do Laboratório de Pesquisa em Avaliação e Medida e pesquisador associado do Instituto de Psicologia da Universidade de Brasília, além de presidente fundador do IBAC. 